\title{
Advancing the Design of Complex Engineered Systems through Multidisciplinary Design Optimization: Report from an NSF Workshop
}

\author{
Timothy W. Simpson* \\ The Pennsylvania State University, University Park, PA \\ Joaquim R. R. A. Martins ${ }^{\dagger}$ \\ University of Michigan, Ann Arbor, MI
}

Multidisciplinary design optimization (MDO) has evolved remarkably since its inception 25 years ago. Despite these advances, the design of complex engineered systems remains a challenge, and many large-scale engineering projects are routinely plagued by exorbitant cost overruns and delays. To gain insight into these challenges, we organized a workshop that gathered 48 people from industry, academia, and government agencies to examine MDO's current and future role in designing complex engineered systems. This presentation summarizes the views of five distinguished speakers on the state of the research along with the discussions from an industry panel of representatives from Boeing, Caterpillar, Ford, NASA Glenn Research Center, and United Technologies Research Center on the state of the practice. This presentation also summarizes the future research topics identified by breakout groups in five key areas: (1) modeling and the design space; (2) metrics, objectives, and requirements; (3) coupling in complex engineered systems; (4) dealing with uncertainty; and (5) people and workflow. Finally, five over-arching themes are offered to advance MDO. First, we need to engage more disciplines outside of engineering and look for opportunities to use MDO outside of its traditional areas. Second, MDO problem formulations must evolve to encompass a wider range of design criteria. Third, we need effective strategies for putting designers "back in the loop" during MDO. Fourth, we need to do a better job of publicizing the successful examples of MDO so that we can improve the "buy in" that is needed to advance MDO in academia, industry, and government agencies. Fifth, we need to better educate our students and practitioners on systems design, optimization and MDO, along with its benefits and drawbacks.

\section{Introduction}

This paper summarizes the presentation that was given at the 8th AIAA Multidisciplinary Design Optimization Specialist Conference, in the special session entitled "MDO Past, Present, Future". A detailed report of the workshop can be found in Ref. [3].

\section{A. Motivation}

Multidisciplinary Design Optimization (MDO) has grown remarkably since its inception 25 years ago.

- Many of its founders are still active in the community

- Research has shifted dramatically as tools have matured

- Commercial MDO tools exist and they are being used

Despite these advances, many question MDOs impact:

- Has MDO transformed industry practice as was intended?

- Why do we still struggle when developing complex systems? For example:

- The Boeing 787 is over two years late and $\$ 10$ billion over budget

- GM lost $\$ 4.3$ billion after resuming operations after bankruptcy

- NASA has cost and schedule overruns with the International Space Station

NSF, DARPA, and others are taking up the challenge of fixing problems plaguing complex systems design.

\footnotetext{
* Professor, Department of Mechanical \& Nuclear Engineering, AIAA Associate Fellow

${ }^{\dagger}$ Associate Professor, Department of Aerospace Engineering, AIAA Senior Member
} 


\section{B. Objectives}

The objectives for the one-day workshop were to:

- Identify and document promising future directions for MDO and related research

- Articulate successful industrial implementations of MDO and identify the challenges with practical implementation of MDO methods and tools

- Explore relationships between complex systems design initiatives and MDO

- Define affinity groups and target specific proposal solicitations and RFPs

The workshop followed the 13th AIAA/ISSMO Multidisciplinary Analysis and Optimization Conference, which celebrated MDO's 25th anniversary. It provided an opportunity to engage academia, industry, and international researchers who attended the conference.

\section{Agenda and Participants}

The agenda for the day was divided into four main parts:

- Presentations by invited speakers from academia and industry (8:30-10 am)

- Industry panel discussion representing multiple sectors (10:30-12:00 noon)

- Breakout group discussions (1:00-3:00 pm)

- Report out and wrap-up (3:00-4:30 pm)

A total of 46 people attended the workshop:

- 26 from academia $(56.5 \%)$

- 12 from industry $(26.1 \%)$

- 8 from government agencies $(17.4 \%)$

- International participants (8) from Canada, Turkey, Portugal, South Korea, and the United Kingdom

All the slides that were presented are available from the workshop website [1]:

http://mdolab.engin.umich.edu/NSF_Workshop_2010

A full report on the workshop with references and detailed discussion has also been published [3].

\section{Invited Speaker Presentations}

The invited speakers were:

- Christina Bloebaum, Program Director, Engineering Design \& Innovation, NSF

- Jaroslaw Sobieski, Distinguished Research Associate, NASA Langley

- Paul Collopy, Director, Value-Driven Design Institute

- Tolga Kurtoglu, Research Scientist, Palo Alto Research Center

- Soundar Kumara, Pearce Professor of Industrial Engineering, Penn State

Some thought-provoking statements included:

"MDO has become everything to everyone and strayed far from its origins of understanding how one designs systems when everything affects everything else."

"It is very difficult for engineers working at the lowest levels of a large-scale engineering system to see the big picture and understand how their decisions impact everything else." 
"Computing power has advanced dramatically in 25 years, yet many people are still trying to solve problems the same way."

"MDO needs to break out of its gilded cage, and find ways to help conceive new designs, not just search the design space defined by the user."

"Research in complex systems and social networks could support MDO by emphasizing (1) modeling, (2) information, and (3) interactions during the design of complex engineered systems."

"MDO is missing out on opportunities in areas such as energy, manufacturing, health care, transportation, financial services, etc."

\section{Industry Panel Discussion}

In this portion of the workshop, industry panelists discussed the state of the practice. The panelists were:

- Evin Cramer, Technical Fellow, The Boeing Company

- Julian Norato, Product Development \& Global Technology, Caterpillar

- Ren-Jye Yang, Senior Technical Leader, Ford Motor Company

- Rubén Del Rosario, Fundamental Aeronautics Program, NASA Glenn

- Ritesh Khire, Senior Research Engineer, United Technologies Research Center

Some of the comments from the industry panelist included:

"MDO is most effective when it becomes a state of mind within the organization"

"The tools should align with the problems" (see Figure 1)

"MDO is 'hot' in industry"

"Projects are underway to make MDO tools more readily available, e.g., NASA's OpenMDAO project at WWW. OpenMDAO. org"

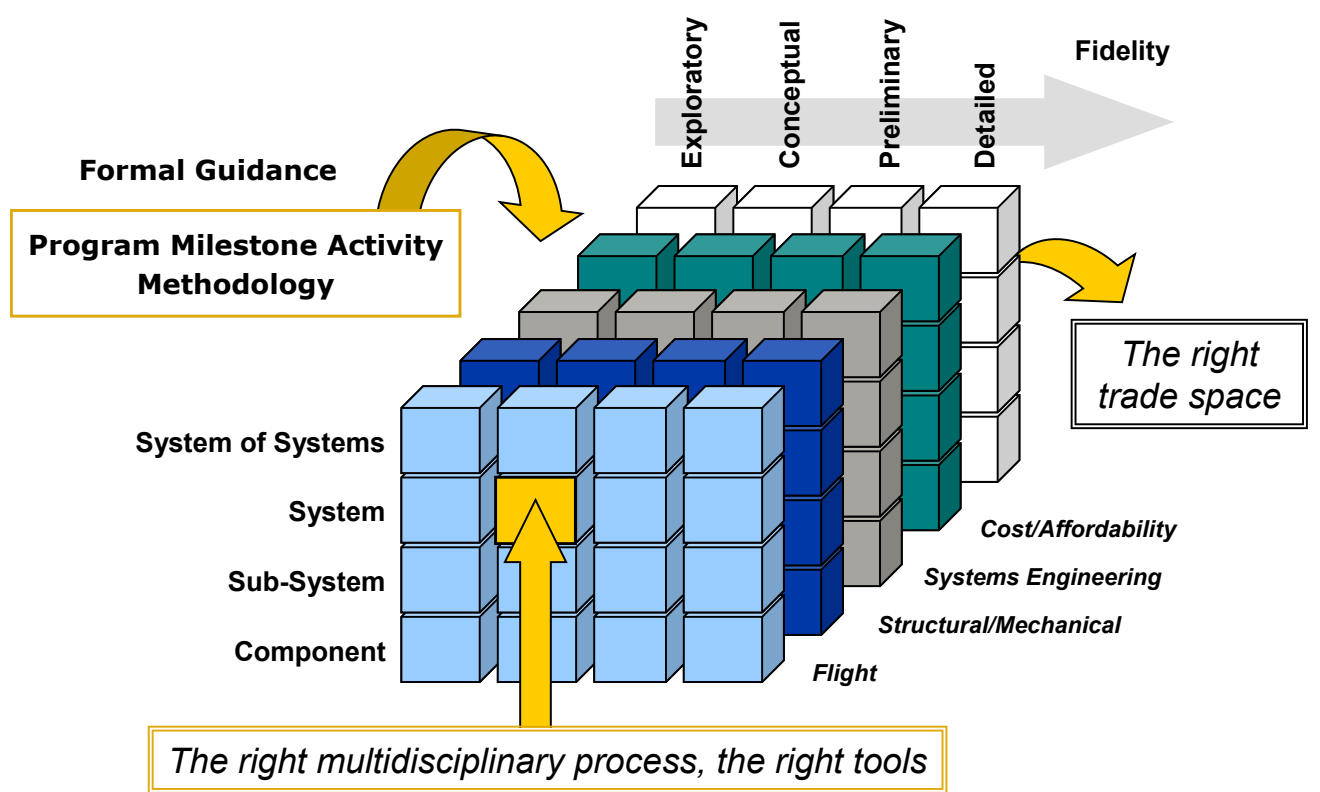

Figure 1: Selecting the right tool for the right problem at the right time (Source: E. Cramer) 


\section{Breakout Discussion}

Before the the speaker presentations and panel discussions, participants were asked to note down questions and important points that they wanted to discuss further in the breakout sessions. Five distinct topics emerged:

1. Modeling and the Design Space: Flexible models and search approaches; managing the design space; capturing emergent behavior; handling problem size and fidelity

2. Metrics, Objectives, and Requirements: Axiomatic measures of complexity, flexibility, etc.; rigorous systems framework; system decomposition and coordination

3. Coupling in Complex Engineered Systems: Flexible MDO systems/frameworks; better understanding of coupling interactions; geometric representations of parts

4. Dealing with Uncertainty: Sources of uncertainty; decision-making under uncertainty; reliability, robustness, safety, durability; risk reduction

5. People and Workflow: Collaborative decision making; teamwork; distributed design; human-in-the-loop approaches; MDO as a state of mind; MDO in education

These group discussions were summarized in slides presented at the workshop, which can be found in the workshop website [1], and more details are reported in Ref. [3].

\section{Recommendations}

The following recommendations were distilled from the the workshop presentations and discussions:

- We must embrace a broader perspective if MDO is to survive

- Integrating disciplinary analyses (e.g., FEA, CFD) is no longer enough

- We need to engage disciplines outside of engineering and look for opportunities to use MDO outside of its traditional areas

- We need new problem formulations that can evolve to encompass a wider range of design criteria

- We need effective strategies for putting designers "back in the loop"

- We need to do a better job of publicizing the successful examples of MDO so that we can improve the "buy in" that is needed to advance MDO in academia, industry, and government agencies

- We need to do a better job of educating students and practitioners about design for a systems perspective, optimization, and MDO, along with their benefits and drawbacks

\section{Related Activities}

Various activities related to the topic took place or have been started since the workshop:

- Special Issue on "Design of Complex Engineered Systems", with J. K. Allen, S. Azarm, and T. W. Simpson, as editors [2]

- 60 papers submitted

- 15 papers accepted for publication

- NSF EFRI 2012 Solicitation: Origami Design for Integration of Self-assembling Systems for Engineering Innovation (ODISSEI)

- NSF and NASA Workshop on Large-Scale Complex Engineered Systems: From Basic Research through Product Realization

- Special session at 8th MDO Specialist Conference, and MDO Special Issue in AIAA Journal, organized by Karen Willcox 


\section{Acknowledgments}

We would like to thank:

- Paul Collopy (Value Driven Design Institute)

- Olivier de Weck (MIT)

- Raphael Haftka (University of Florida)

- Andrew March (MIT)

- Christopher Mattson (Brigham Young University)

- Achille Messac (Syracuse University)

- Jaroslaw Sobieski (NASA Langley Research Center)

- Irem Tumer (Oregon State University)

- Karen Willcox (MIT)

- All workshop participants

This workshop was supported by the National Science Foundation under Collaborative Grants CMMI-1042397 \& CMMI-104270. Any opinions, findings, and conclusions or recommendations in this paper are those of the authors and workshop participants, and do not necessarily reflect the views of the National Science Foundation.

\section{References}

[1] September 2010. URL http://mdolab.engin.umich.edu/NSF_Workshop_2010.

[2] J. K. Allen, S. Azarm, , and T. W. Simpson, editors. Special issue of the Journal of Mechanical Design — "Design of Complex Engineered Systems”, volume 133, issue 10. October 2011.

[3] T. W. Simpson and J. R. R. A. Martins. Multidisciplinary design optimization for complex engineered systems design: Report from an NSF workshop. Journal of Mechanical Design, 133(10):101002, October 2011. doi:10.1115/1.4004465. 